\title{
Increased dose of re-irradiation therapy or combined chemotherapy to improve survival of local recurrent esophageal squamous cell carcinoma after radiotherapy
}

\section{Xun Wu ( $\nabla$ yjs2016nsmc@sina.com)}

Chengdu Fifth people's hospital

\section{Xingsheng Hu}

The Second Xiangya Hospital of Central South University

Junru Chen

Sichuan Cancer Hospital and Research Institute

\section{Lang He}

Chengdu Fifth People's Hospital

\section{Research}

Keywords: esophageal squamous cell carcinoma, recurrence, salvage chemoradiation therapy, salvage radiation therapy

Posted Date: August 14th, 2020

DOI: https://doi.org/10.21203/rs.3.rs-56036/v1

License: (c) (1) This work is licensed under a Creative Commons Attribution 4.0 International License.

Read Full License 


\section{Abstract}

Introduction: This study aimed to explore the optimal treatment for locally recurrent esophageal squamous cell carcinoma (LRESCC) by analyzing the results of different doses of re-irradiation therapy after radical (chemo) radiotherapy.

Methods: We retrospectively analyzed 125 patients with LRESCC after initial radiotherapy. According to radiotherapy dose, 58 patients were assigned to the low-dose (LD) group (50-54 Gy) and 67 to the highdose (HD) group (55-60 Gy). We recorded the response rate (complete + partial response), 1-, 2- and 3year survival rates, and toxicity in the two groups. The effects of different radiotherapy doses and combination chemotherapy on the survival of LRESCC were analyzed.

Results: After re-irradiation, the 1-, 2- and 3-year survival rates were $48.3 \%, 24.1 \%$ and $10.3 \%$ in the LD group and $61.2 \%, 34.3 \%$ and $19.4 \%$ in the HD group $(P<0.05)$, respectively. The median survival time of patients receiving radiotherapy alone was 9 months in the LD group and 15 months in the HD group $(P<0.05)$. The survival rate of patients treated with chemoradiotherapy was higher than that of patients treated with radiotherapy alone in the LD group, but in the HD group, chemoradiotherapy showed no advantage compared with radiotherapy alone. The most common toxicity was esophagitis, and the incidence was higher in the HD group than in the LD group (68.7\% vs $58.6 \%, P \llbracket 0.05$ ).

Conclusion: Increasing the dose of re-irradiation therapy or chemotherapy can improve the efficacy of reirradiation therapy for patients with LRESCC after radiotherapy.

\section{Introduction}

Esophageal squamous cell carcinoma (ESCC) is the most common esophageal malignant tumor in China. It is moderately sensitive to radiation. Comprehensive treatment based on radiotherapy or surgery is its main treatment. Unfortunately, the local recurrence rate of esophageal cancer is as $40 \%-60 \%$ after radiotherapy ${ }^{[1]}$. To date, there is still no consensus on the retreatment of local recurrent ESCC (LRESCC) after (chemo)radiotherapy. Studies have shown that salvage esophagectomy is effective in treating locally recurrent esophageal cancer [2, 3]. Chen et al. reported that re-irradiation can achieve similar efficacy to salvage surgery ${ }^{[4]}$. In addition, fibrosis of the tissue surrounding the tumor bed after radiotherapy increases the difficulty of surgery and perioperative risk. Therefore, even carefully selected patients should be cautiously considered for surgery $[5,6]$.

There are still many problems in the re-irradiation of LRESCC. Radiotherapy dose is an important factor affecting efficacy. There is no consensus on the implementation of re-irradiation dose. Chemotherapy is often used as a systemic treatment, but in the case of repeat radiotherapy for LRESCC, the role of combined chemotherapy is not clear. Therefore, the purpose of present study was to evaluate the efficacy and toxicity of different radiotherapy doses and combined chemotherapy with re-irradiation therapy for LRESCC. 


\section{Patients And Methods}

\section{Patients and clinical features}

We retrospectively selected 207 patients with LRESCC who underwent re-irradiation therapy in the Chengdu Fifth People's Hospital between January 2012 and December 2016. The inclusion criteria were: 1) non-recurrence interval $>6$ months after the end of initial radiotherapy; 2 ) pathological examination, imaging or gastroscopy confirmed the recurrence of primary esophageal cancer with or without local lymph node metastasis, or only local lymph node metastasis (supraclavicular fossa, mediastinal, esophageal, or para-aortic lymph nodes); 3) re-irradiation therapy dose 50-60 Gy; 4) Eastern Cooperative Oncology Group (ECOG) score 0-2, and good function of heart, liver, kidneys, lungs, and bone marrow hematopoiesis. The exclusion criteria were: 1) distant organ metastasis, tumor in other organs, or incomplete information or hyper-fractionated radiotherapy; 2 ) a history of surgical resection of esophageal cancer. Finally, 125 patients were included, 84 men and 41 women with a median age of 68 years (range 50-89 years). This study was approved by the Ethics Committee of Chengdu Fifth People's Hospital.

\section{Treatment and follow-up}

In the initial treatment, two-dimensional radiotherapy, three-dimensional conformal radiotherapy (3D-CRT) or intensity modulated radiotherapy (IMRT) was used for radical radiotherapy for the 125 patients, with a median dose of 60 Gy (50-66 Gy). All patients did not undergo surgery. Most patients received chemotherapy regimens containing cisplatin, paclitaxel or fluorouracil. After initial treatment, gastroscopy, esophageal barium meal radiography, or enhanced computed tomography (CT) was used to evaluate the efficacy, and complete response or partial response was achieved in all patients.

All patients were re-irradiation with 3D-CRT or IMRT with 1.8-2.0 Gy per fraction, 5 days/week. Gross tumor volume (GTV) was observed on esophageal barium meal examination and CT images. GTV of the metastatic lymph nodes (GTVnd) included enlarged lymph nodes with a short diameter $>10 \mathrm{~mm}$, or positron emission tomography-CT examination suggested metastatic lymph nodes. Planning target volume (PTV) was formed by extending $0.5-1.0 \mathrm{~cm}$ radially from GTV, $1.0-2.0 \mathrm{~cm}$ above and below GTV, or $0.5 \mathrm{~cm}$ outside GTVnd in all directions. The lymphatic drainage area was not irradiated prophylactically. Dose limitation of normal tissue or organ was: bilateral lungs $\mathrm{V} 20<25 \%$, spinal cord Dmax $<20 \mathrm{~Gy}$, and mean radiation dose to the heart $<30 \mathrm{~Gy}$.

The overall survival (OS) was defined as time from the beginning of retreatment until patient death or last follow-up. Efficacy was evaluated at the end of retreatment and 1 month after the end of retreatment. Evaluation methods included endoscopy, tumor markers, esophageal barium meal, and enhanced CT. The response rate (RR) was defined as the percentage of patients with complete response and partial response in the total number of cases. Therapeutic evaluation criteria refers to response evaluation criteria in solid tumors (RECIST 1.1). Toxicity was graded by the Common Terminology Criteria for Adverse Events (CTCAE v4.0). According to the re-irradiation dose, 58 patients were assigned to the low- 
dose (LD) group (50-54 Gy, median dose $50 \mathrm{~Gy}$ ) and 67 to the high-dose (HD) group (55-60 Gy, median dose $60 \mathrm{~Gy})$.

\section{Statistical analysis}

Statistical analysis of the database was performed using SPSS version 23.0 (IBM Corporation, Armonk, NY, USA). The continuous and categorical variables in the two treatment groups were compared by $t$-test and chi-square test, respectively. OS was calculated from the first day of retreatment to the patient's death or last follow-up. The survival rates were calculated by Kaplan-Meier method, and the difference in survival curves was compared by log-rank method. Cox's proportional hazards regression model was used to determine the effect of univariate and multiple factors on survival. Non-recurrence interval (NRI) was defined as the time between the beginning of initial treatment and diagnosis of recurrence. Twosided $P<0.05$ was considered to indicate statistical significance.

\section{Results}

The clinical baseline characteristics of the two groups of patients are shown in Table 1. The median time to NRI in all patients was 21 months (range 8-201 months). Sixty-eight (54.4\%) patients relapsed within 2 years after initial treatment. The last follow-up time was December 2019 with a median follow-up of 19 months (range 4-65 months). At the end of the follow-up, two patients survived in the LD group, seven survived in the HD group; six of the nine patients had a second recurrence, and three did not have a second recurrence or metastasis. There were 67 cases of primary recurrence (PR), 33 cases of primary recurrence accompanied by local lymph node recurrence (PR $+L N R)$ and 25 cases of local lymph node recurrence (LNR). Sixty-one of the 125 patients received concurrent or 1-4 sequential cycles of chemotherapy, which included either single or double-drug regimens containing paclitaxel, platinum or fluorouracil. 
Table 1

Patients' characteristics $(n=125)$

\begin{tabular}{|c|c|c|c|c|}
\hline Characteristics & LD group(\%) & HD group(\%) & $\chi^{2} / t$ & $P$ \\
\hline \multicolumn{5}{|l|}{ Gender } \\
\hline Male & $35(60.3)$ & 49(73.1) & 2.307 & 0.129 \\
\hline Female & 23(39.7) & $18(26.9)$ & & \\
\hline \multicolumn{5}{|l|}{ Age } \\
\hline Mean \pm SD & $69.34 \pm 8.13$ & $68.18 \pm 7.53$ & 0.832 & 0.407 \\
\hline \multicolumn{5}{|l|}{ ECOG } \\
\hline $0-1$ & $48(82.8)$ & $54(80.6)$ & 0.097 & 0.756 \\
\hline 2 & 10(17.2) & $13(19.4)$ & & \\
\hline \multicolumn{5}{|c|}{ Recurrence pattern } \\
\hline PR & $35(60.3)$ & $32(47.8)$ & 1.614 & 0.446 \\
\hline LN & 11(19.0) & 14(20.9) & & \\
\hline$P R+L N$ & $12(20.7)$ & 21(31.3) & & \\
\hline \multicolumn{5}{|c|}{ Initial radiation dose (Gy) } \\
\hline Mean dose & $58.69 \pm 3.10$ & $59.22 \pm 2.76$ & 1.01 & 0.310 \\
\hline \multicolumn{5}{|l|}{ NRI (months) } \\
\hline$\leq 24$ & $36(62.1)$ & $32(47.8)$ & 2.57 & 0.109 \\
\hline$\otimes 24$ & $22(37.9)$ & $35(52.2)$ & & \\
\hline \multicolumn{5}{|c|}{ Re-radiotherapy technology } \\
\hline 3D-CRT & $25(43.1)$ & $34(50.7)$ & 0.73 & 0.393 \\
\hline IMRT & $33(56.9)$ & $33(49.3)$ & & \\
\hline \multicolumn{5}{|l|}{ Chemotherapy } \\
\hline Yes & $25(43.1)$ & $36(53.7)$ & 1.405 & 0.236 \\
\hline No & $33(56.9)$ & $31(46.3)$ & & \\
\hline
\end{tabular}

\section{Treatment outcomes}

The 1-, 2- and 3-year survival rates for the whole cohort were $56.8 \%, 29.6 \%$ and $15.2 \%$, with a median survival time of 14 months. The 1-, 2- and 3-year survival rates in the LD group were $48.3 \%, 24.1 \%$ and 
$10.3 \%$, respectively, and the median survival time was 11 months ( $95 \%$ confidence interval (Cl): 8.51413.486 months). The 1-, 2- and 3-year survival rates in the HD group were $61.2 \%, 34.3 \%$ and $19.4 \%$, respectively, and the median survival time was 18 months ( $95 \% \mathrm{Cl}$ : 12.276-23.724 months). Patients in the HD group showed a better survival advantage compared to the LD group (Fig. 2). After retreatment, the RR was $87.9 \%$ in the HD group (58/66), which was higher than $76.8 \%$ in the LD group (43/56), the difference was not significant $(P=0.065)$. The 3-year locoregional control rates in the LD group and that in the HD group were $3.4 \%$ and $14.9 \%$, respectively (Fig. 1). There was a significant difference between local control rates in the two groups (log-rank test, $p=0.039$ ).

In stratified analysis, median survival time was 15 months (95\% Cl: 7.365-22.635 months) in 31 patients in the HD group who received radiotherapy alone. This was superior to median survival of 9 months (95\% Cl: 7.124-10.876 months) in 33 patients in the LD group who received radiotherapy alone (Fig. 3A). The difference between the two groups was significant $(P=0.009)$. However, in the stratified analysis of patients received chemotherapy in the two groups, patients in the HD group did not show survival benefit (Fig. 3B).

In subgroup analysis, we found that chemotherapy had a significant effect on survival in the LD group. In the LD group, patients with chemoradiotherapy showed superior median survival than patients with radiotherapy alone (14 months, 95\% Cl: 12.378-15.622 months vs 9 months, 95\% Cl: 7.124-10.876 months) (Fig. 4A). The difference was significant. However, chemotherapy did not continue to increase the survival advantage of patients in the HD group (Fig. 4B).

\section{Cox regression analysis for overall sample}

We conducted a multivariate analysis in order to examined whether the dose of re-irradiotherapy was a factor affecting the prognosis of patients. The results of univariate and multivariate analyses for OS were summarized in Table 3. ECOG of $0-1(P=0.048)$, age $₫ 70$ years $(P=0.028)$, NRI $\varangle 24$ months $(P=0.001)$ and re-irradiation dose of 55-60 Gy $(P=0.013)$ were associated with favorable OS by Cox analysis. 
Table 3

Prognostic factors for patient survival

\begin{tabular}{|c|c|c|c|c|c|}
\hline \multirow[t]{2}{*}{ Variables } & & \multicolumn{2}{|l|}{ Univariate } & \multicolumn{2}{|l|}{ Multivariate } \\
\hline & & $\mathrm{HR}(95 \% \mathrm{Cl})$ & $\mathbf{P}$ & $\mathrm{HR}(95 \% \mathrm{Cl})$ & $\mathbf{P}$ \\
\hline gender & & $0.758(0.505-1.138)$ & 0.181 & & \\
\hline male & 84 & & & & \\
\hline female & 41 & & & & \\
\hline age (years) & & $1.519(1.046-2.208)$ & 0.028 & $1.472(1.010-2.144)$ & 0.044 \\
\hline$\otimes 70$ & 71 & & & & \\
\hline$\geq 70$ & 54 & & & & \\
\hline ECOG & & $1.593(1.004-2.527)$ & 0.048 & $1.641(1.027-2.621)$ & 0.038 \\
\hline $0-1$ & 102 & & & & \\
\hline 2 & 23 & & & & \\
\hline Recurrence pattern & & $1.149(0.915-1.444)$ & 0.232 & & \\
\hline PR & 67 & & & & \\
\hline LN & 25 & & & & \\
\hline$P R+L N$ & 33 & & & & \\
\hline NRI (months) & & $0.507(0.346-0.745)$ & 0.001 & $0.484(0.328-0.713)$ & $<0.001$ \\
\hline$\leq 24$ & 68 & & & & \\
\hline$₫ 24$ & 57 & & & & \\
\hline chemotherapy & & $0.758(0.526-1.094)$ & 0.139 & & \\
\hline yes & 61 & & & & \\
\hline no & 64 & & & & \\
\hline re-irradiation dose & & $0.626(0.432-0.908)$ & 0.013 & $0.638(0.439-0.927)$ & 0.018 \\
\hline 50-54Gy & 58 & & & & \\
\hline $55-60 G y$ & 67 & & & & \\
\hline
\end{tabular}

\section{Toxicity}

Esophagitis was the most common toxicity in the two groups. The incidence of esophagitis was $58.6 \%$ in the LD group and $68.7 \%$ in the HD group. The difference was not significant $(P=0.868)$. Other toxicity 
included hematological toxicity, the difference was significant $(p=0.004)$ and gastrointestinal reaction, the difference was not significant $(p=0.732)$. Rare adverse effects included radiation-induced pneumonitis, radiation tracheitis and skin reaction (Table 2). Gastrointestinal reactions include nausea, vomiting and loss of appetite. Hematological toxicity was mainly reduced white blood cells and neutrophils, and anemia. After symptomatic treatment, all patients completed radiotherapy. Eleven patients developed severe treatment-related toxicity, including esophageal perforation/fistula and bleeding. The NRI of most of the 11 patients (9 / 11) was $\leq 24$ months. After six months of retreatment, two patients in each group had severe esophageal stenosis and underwent esophageal dilatation.

Table 2

Toxic effects in the two groups (n)

\begin{tabular}{|lllllll|}
\hline Toxicity & \multicolumn{3}{c}{ LD group } & \multicolumn{4}{c|}{ HD group } \\
\cline { 2 - 8 } & $\mathbf{1}$ & $\mathbf{2}$ & $\mathbf{3}$ & $\mathbf{1}$ & $\mathbf{2}$ & $\mathbf{3}$ \\
\hline Esophagitis & 19 & 13 & 2 & 29 & 14 & 3 \\
\hline Hematologic toxicity & 23 & 11 & 6 & 12 & 19 & 8 \\
\hline Gastrointestinal reactions & 6 & 5 & 3 & 12 & 7 & 3 \\
\hline Radiation-induced pneumonitis & 6 & 0 & 0 & 7 & 1 & 0 \\
\hline Tracheitis & 2 & 0 & 0 & 1 & 1 & 0 \\
\hline Skin reaction( $\geq 2)$ & 3 & & & 4 & & \\
\hline Bleeding & 3 & & & & 1 & \\
\hline Esophageal perforation/fistula & 5 & & & & 2 & \\
\hline esophageal stenosis & 2 & & & 2 & & \\
\hline
\end{tabular}

\section{Discussion}

External beam radiotherapy plays an important role in relieving the symptoms of esophageal cancer and maintaining quality of life of patients, but its effects are limited. Most patients have recurrence or metastasis within 2 years after radiotherapy $[4,7,8]$. Without active intervention, the survival and prognosis of patients with disease progression are often poor, and most die within 1 year [9]. Although many researchers are actively exploring the best salvage treatment for LRESCC, there is still no consensus. Many studies have confirmed the positive role of chemotherapy in the initial treatment of ESCC [10], but the role of chemotherapy in the re-irradiation therapy of ESCC is not clear. The clinical efficacy of recurrent ESCC salvage radiotherapy is significantly affected by the radiation dose [11]. Up to date, the optimal dose of salvage radiotherapy is still unclear. 
Recently, a study of the effect of dose-escalated radiotherapy on ESCC showed that, in patients who achieved complete response, radiotherapy dose was increased to $>59.4$ Gy after standard concurrent chemoradiotherapy (50.4 Gy), which significantly improved local control, 5-year recurrence-free survival rate and 5-year OS rate [12]. Previously, some studies have indicated that the survival rate of LRESCC can be significantly prolonged when the dose of re-irradiation is $>50 \mathrm{~Gy}[4,13]$. Kobayashi et al. pointed out that 60 Gy was an appropriate salvage dose for LRESCC after surgery [14]. Another study from Japan pointed out that patients with LRESCC with a radiation history should be given higher doses of radiotherapy [15]. These studies suggest that increasing the dose of radiotherapy for recurrent esophageal cancer may be beneficial to prolong survival. However, there is no report on whether higher doses of re-irradiation between 50 and 60 Gy would benefit more patients with LRESCC with radiation history.

This study is believed to be the first to compare the effects of $50 \mathrm{~Gy}$ and $60 \mathrm{~Gy}$ re-irradiation dose on the survival of patients with LRESCC with radiation history. Our results showed that most patients $(68 / 125$, $54.4 \%$ ) had recurrence within 2 years after initial (chemo)radiotherapy, which was consistent with previous reports $[4,8,13,16]$. Jingu et al. reported that the 3-year survival rate and median survival time of 33 patients with LRESCC receiving $60 \mathrm{~Gy}$ (range, 18-70 Gy) re-radiotherapy were $17.8 \%$ and 16 months, respectively [15]. Hong et al. reported that the median survival time of recurrent ESCC patients receiving 50 Gy (range, 21-70 Gy) re-radiotherapy was 10 months[17]. This is close to our survival results. In the present study, the 1-and 3-year survival rates and median survival time were $61.2 \%$ and $19.4 \%$ and 18 months in the HD group, respectively. The 1- and 3-year survival rate and median survival time were $48.3 \%$ and $10.3 \%$ and 11 months in the LD group, respectively. Radiotherapy dose is an important factor affecting survival, which was clearly demonstrated in our results. The median survival time of patients receiving radiation alone in the HD group was 15 months, which was significantly higher than 9 months in the LD group. Increasing the dose of radiation showed an encouraging outcome. Our results are also better compared with those of other studies $[4,13,15]$. The reason may be that higher doses of radiation were more beneficial for tumor control. Therefore, we recommend higher doses of radiation for patients with recurrent ESCC after radiotherapy.

Few studies have addressed the role of chemotherapy in re-radiotherapy for recurrent ESCC. Chen et al. reported that the 1-, 2- and 3-year survival rates of 36 LRESCC patients receiving re-irradiation with concurrent chemotherapy (paclitaxel + cisplatin) were $51.7 \%, 21.4 \%$ and $12.2 \%$, respectively [4]. Katano et al. reported that the median survival time of six patients who underwent concurrent chemotherapy with nedaplatin and tegafur with re-radiotherapy was 13.6 months[6]. As of now, the role of chemotherapy in re-radiotherapy for recurrent ESCC is still unclear. In subgroup analysis, our results showed that chemotherapy combined with radiotherapy could increase survival rate. In the LD group, the median survival time for chemoradiotherapy was 14 months, which was consistent with the results reported by Katano et al. [6]. However, in the HD group, chemoradiotherapy did not show better efficacy than radiotherapy alone, and other studies have reported the same [17]. Our results suggest that chemotherapy can increase the survival rate of re-radiotherapy for ESCC, especially when patients are exposed to lower 
radiation doses. This may be related to the fact that chemotherapy increases the tumor control rate at low rather than high dose of radiotherapy.

In this study, the most common toxicity of the whole cohort was esophagitis. Although the incidence of esophagitis in the HD group was higher than in the LD group ( $68.7 \%$ vs $58.6 \%)$, the difference was not significant. A previous study showed that the incidence of severe acute esophagitis in patients with thoracic radiotherapy was $15-25 \%[18]$. Grade 3 or higher acute esophagitis was rare in the two groups in this study. Hematological toxicity and gastrointestinal reactions were also common, and only hematologic toxicity was significant between the two groups. In this study, the incidence of radiation pneumonitis was lower than in previous studies[19]. Grade 1-2 radiation pneumonitis occurred in six (10.3\%) and eight (11.9\%) patients in the LD and HD groups, respectively, and the symptoms was controllable. We recorded eleven patients with severe treatment-related toxicity, including bleeding, esophageal perforation, and esophageal fistula, and eight of eleven were found in the LD group. This may be related to the NRI of $\leq 24$ months in most patients $(36 / 58,62.1 \%)$ in the LD group. Current results indicated that a higher re-irradiation dose was safe.

\section{Conclusion}

We recommend that a higher re-irradiation dose (55-60 Gy) for LRESCC patients with radiation history may be more conducive to long-term survival, and toxicity is tolerable. Re-irradiation combined with chemotherapy can improve survival rate of patients, especially those receiving lower doses of reirradiation (50-54 Gy). For those patients with a short recurrence time (NRI $\leq 24$ months), we should be vigilant for serious toxicity that may lead to rapid death during re-irradiotherapy.

\section{Abbreviations}

LRESCC: locally recurrent esophageal squamous cell carcinoma; LD group: low-dose group; HD group: high-dose group; ESCC: Esophageal squamous cell carcinoma; ECOG: Eastern Cooperative Oncology Group Performance Status; 3D-CRT: three-dimensional conformal radiotherapy; IMRT: intensity modulated radiotherapy; CT: computed tomography; RR: response rate; GTV: Gross tumor volume; GTVnd: Gross tumor volume of the metastatic lymph nodes; PTV: Planning target volume; OS: overall survival; RECIST 1.1: Response Evaluation Criteria in Solid Tumors Version 1.1; CTCAE v4.0: Common Terminology Criteria for Adverse Events version 4.0; NRI: Non-recurrence interval; LNR: local lymph node recurrence; PR: primary recurrence; $\mathrm{Cl}$ : confidence interval

\section{Declarations}

\section{Ethical Approval and Consent to participate}

This study was approved by the Ethics Committee of Chengdu Fifth People's Hospital, Chengdu, China.

\section{Consent for publication}


Not applicable.

Availability of data and materials

All data generated or analyzed during this study are included in this article.

Competing interests

The authors declared that they had no competing interests.

Funding

None.

Authors' contributions

XUN WU and JUNRU CHEN participated in research design. Acquisition of the data was performed by XINGSHENG HU and XUEMEI YANG. Evaluation of the images was conducted by XINGSHENG HU and LANG HE. The manuscript written by XUN WU. All authors read and approved the final manuscript.

\section{Acknowledgements}

We thank Professor Guangming Li for his guidance on the design of this study.

\section{References}

1. Pennathur A, Gibson MK, Jobe BA, Luketich JD: Oesophageal carcinoma. The Lancet 2013, 381(9864):400-412.

2. Sudo K, Xiao L, Wadhwa R, Shiozaki H, Elimova E, Taketa T, Blum MA, Lee JH, Bhutani MS, Weston B et al: Importance of surveillance and success of salvage strategies after definitive chemoradiation in patients with esophageal cancer. Journal of clinical oncology : official journal of the American Society of Clinical Oncology 2014, 32(30):3400-3405.

3. Takeuchi H, Saikawa Y, Oyama T, Ozawa S, Suda K, Wada N, Takahashi T, Nakamura R, Shigematsu $\mathrm{N}$, Ando $\mathrm{N}$ et al: Factors influencing the long-term survival in patients with esophageal cancer who underwent esophagectomy after chemoradiotherapy. World journal of surgery 2010, 34(2):277-284.

4. Chen Y, Lu Y, Wang Y, Yang H, Xia Y, Chen M, Song H, Li T, Li D, Wang J et al: Comparison of salvage chemoradiation versus salvage surgery for recurrent esophageal squamous cell carcinoma after definitive radiochemotherapy or radiotherapy alone. Diseases of the esophagus : official journal of the International Society for Diseases of the Esophagus 2014, 27(2):134-140.

5. D'Journo XB, Thomas PA: Current management of esophageal cancer. Journal of thoracic disease 2014, 6 Suppl 2:S253-264.

6. Katano A, Yamashita H, Nakagawa K: Re-irradiation of locoregional esophageal cancer recurrence following definitive chemoradiotherapy: A report of 6 cases. Molecular and clinical oncology 2017, 
7(4):681-686.

7. Stahl M, Stuschke M, Lehmann N, Meyer HJ, Walz MK, Seeber S, Klump B, Budach W, Teichmann R, Schmitt $\mathrm{M}$ et al: Chemoradiation with and without surgery in patients with locally advanced squamous cell carcinoma of the esophagus. Journal of clinical oncology : official journal of the American Society of Clinical Oncology 2005, 23(10):2310-2317.

8. Amini A, Ajani J, Komaki R, Allen PK, Minsky BD, Blum M, Xiao L, Suzuki A, Hofstetter W, Swisher S et al: Factors associated with local-regional failure after definitive chemoradiation for locally advanced esophageal cancer. Annals of surgical oncology 2014, 21(1):306-314.

9. Kim YS, Lee CG, Kim KH, Kim T, Lee J, Cho Y, Koom WS: Re-irradiation of recurrent esophageal cancer after primary definitive radiotherapy. Radiation oncology journa/2012, 30(4):182-188.

10. Shridhar R, Almhanna K, Meredith KL, Biagioli MC, Chuong MD, Cruz A, Hoffe SE: Radiation therapy and esophageal cancer. Cancer Control 2013, 20(2):97-110.

11. Fakhrian K, Gamisch N, Schuster T, Thamm R, Molls M, Geinitz H: Salvage radiotherapy in patients with recurrent esophageal carcinoma. Strahlentherapie und Onkologie : Organ der Deutschen Rontgengesellschaft [et al] 2012, 188(2):136-142.

12. Zhang W, Luo Y, Wang X, Han G, Wang P, Yuan W, Dai SB: Doseescalated radiotherapy improved survival for esophageal cancer patients with a clinical complete response after standard-dose radiotherapy with concurrent chemotherapy. Cancer Manag Res 2018, 10:2675-2682.

13. Zhou ZG, Zhen CJ, Bai WW, Zhang P, Qiao XY, Liang JL, Gao XS, Wang SS: Salvage radiotherapy in patients with local recurrent esophageal cancer after radical radiochemotherapy. Radiation oncology (London, England) 2015, 10:54.

14. Kobayashi R, Yamashita H, Okuma K, Shiraishi K, Ohtomo K, Nakagawa K: Salvage radiation therapy and chemoradiation therapy for postoperative locoregional recurrence of esophageal cancer. Diseases of the esophagus : official journal of the International Society for Diseases of the Esophagus 2014, 27(1):72-78.

15. Jingu K, Niibe Y, Yamashita H, Katsui K, Matsumoto T, Nishina T, Terahara A: Re-irradiation for oligorecurrence from esophageal cancer with radiotherapy history: a multi-institutional study. Radiation oncology (London, England) 2017, 12(1):146.

16. Ishihara R, Yamamoto S, lishi H, Takeuchi Y, Sugimoto N, Higashino K, Uedo N, Tatsuta M, Yano M, Imai A et al: Factors predictive of tumor recurrence and survival after initial complete response of esophageal squamous cell carcinoma to definitive chemoradiotherapy. International journal of radiation oncology, biology, physics 2010, 76(1):123-129.

17. Hong L, Huang YX, Zhuang QY, Zhang XQ, Tang LR, Du KX, Lin XY, Zheng BH, Cai SL, Wu JX et al: Survival benefit of re-irradiation in esophageal Cancer patients with Locoregional recurrence: a propensity score-matched analysis. Radiation oncology (London, England) 2018, 13(1):171.

18. Werner-Wasik M, Pequignot E, Leeper D, Hauck W, Curran W: Predictors of severe esophagitis include use of concurrent chemotherapy, but not the length of irradiated esophagus: a multivariate analysis 
of patients with lung cancer treated with nonoperative therapy. International journal of radiation oncology, biology, physics 2000, 48(3):689-696.

19. Li CC, Chen CY, Chien CR: Comparison of intensity-modulated radiotherapy vs 3-dimensional conformal radiotherapy for patients with non-metastatic esophageal squamous cell carcinoma receiving definitive concurrent chemoradiotherapy: A population-based propensity-score-matched analysis. Medicine (Baltimore) 2018, 97(22):e10928.

Figures

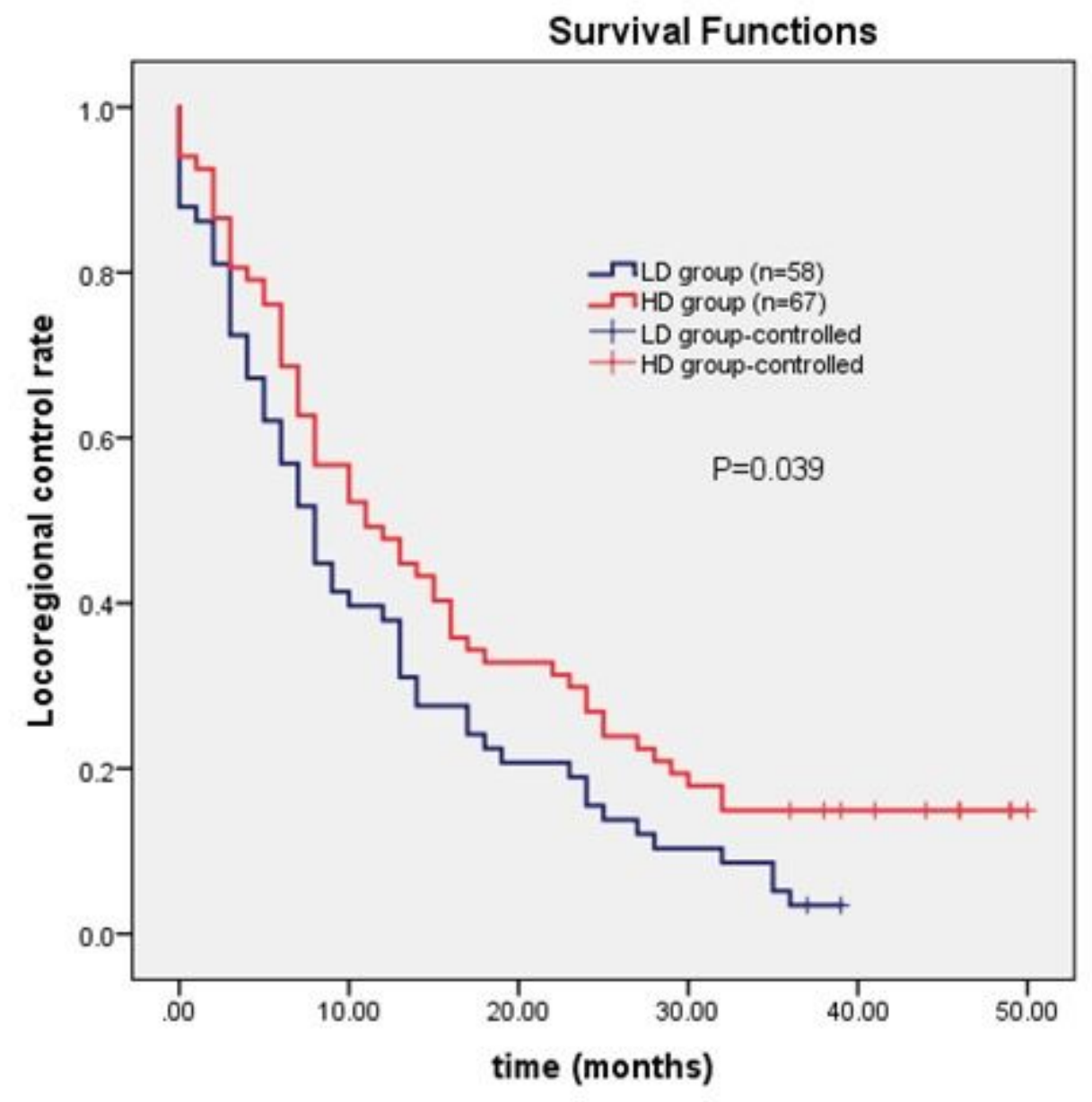

Figure 1

There was a significant difference between locoregional control rate in the LD group and HD group (Kaplan-Meier method) 


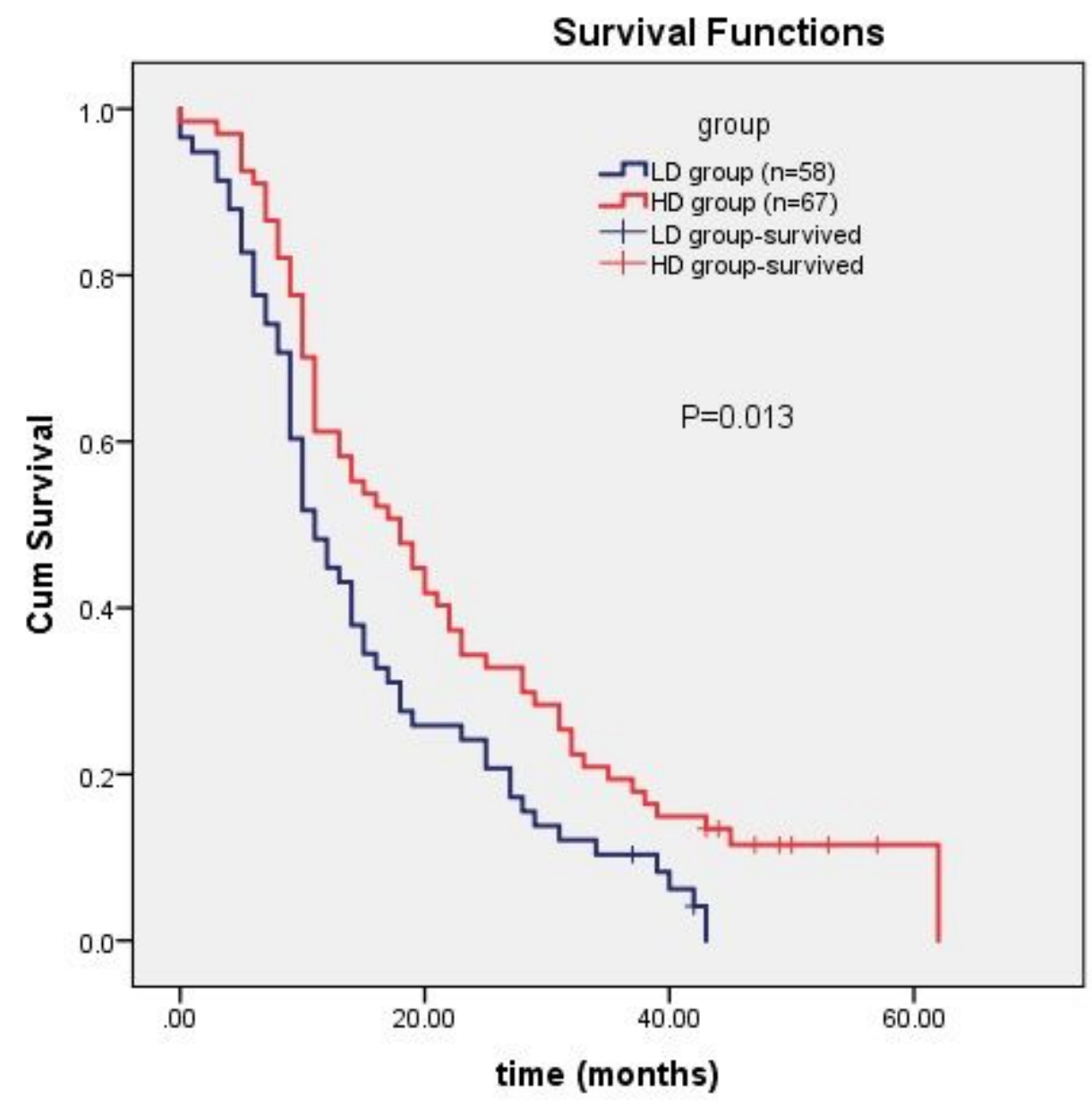

Figure 2

Survival curve of patients in the low-dose and high-dose groups after re-irradiation therapy. 


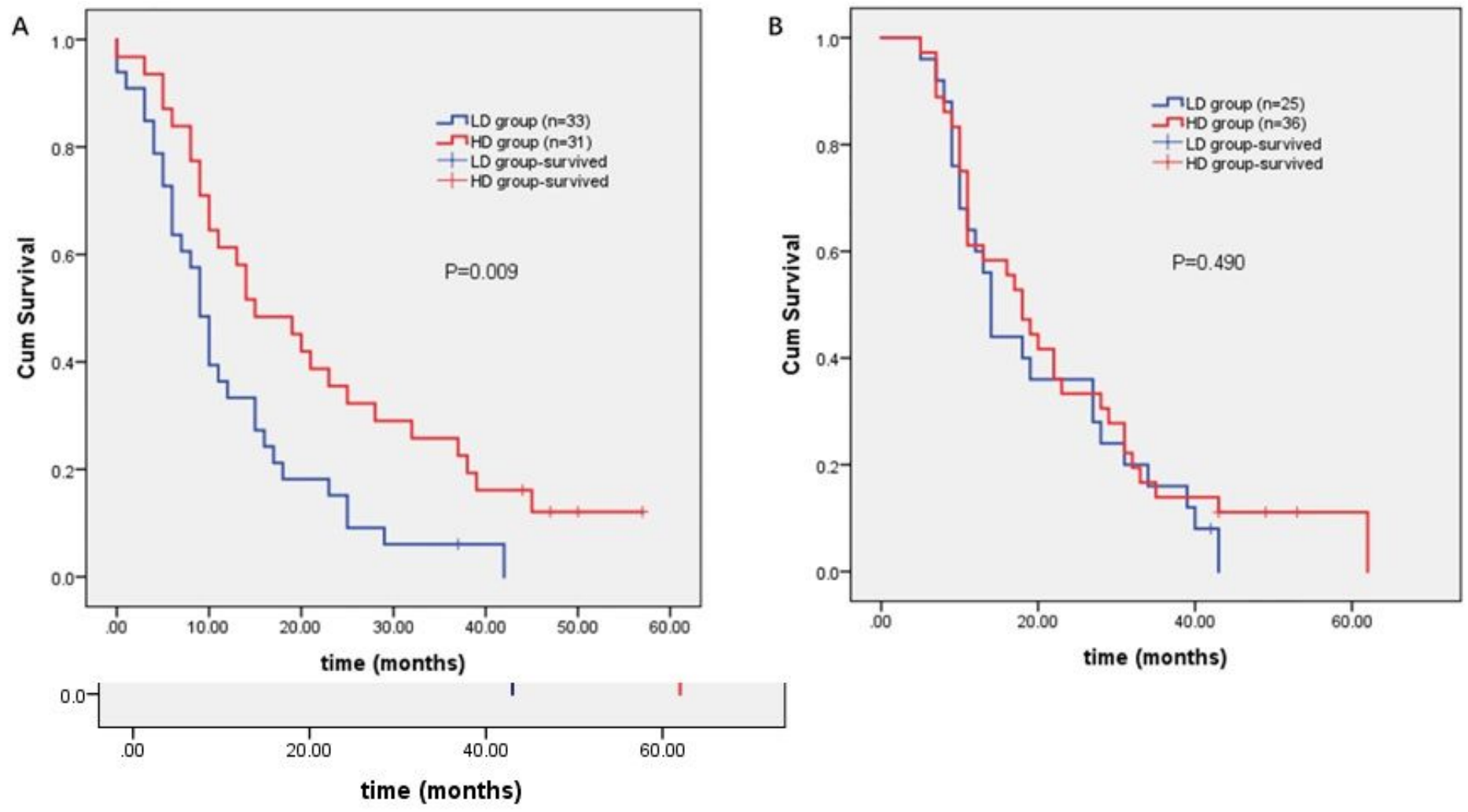

Figure 3

A Survival curve of patients in the low-dose and high-dose groups after with re-irradiotherapy alone. B Survival curve of patients in low-dose group and high-dose group with chemoradiotherapy.
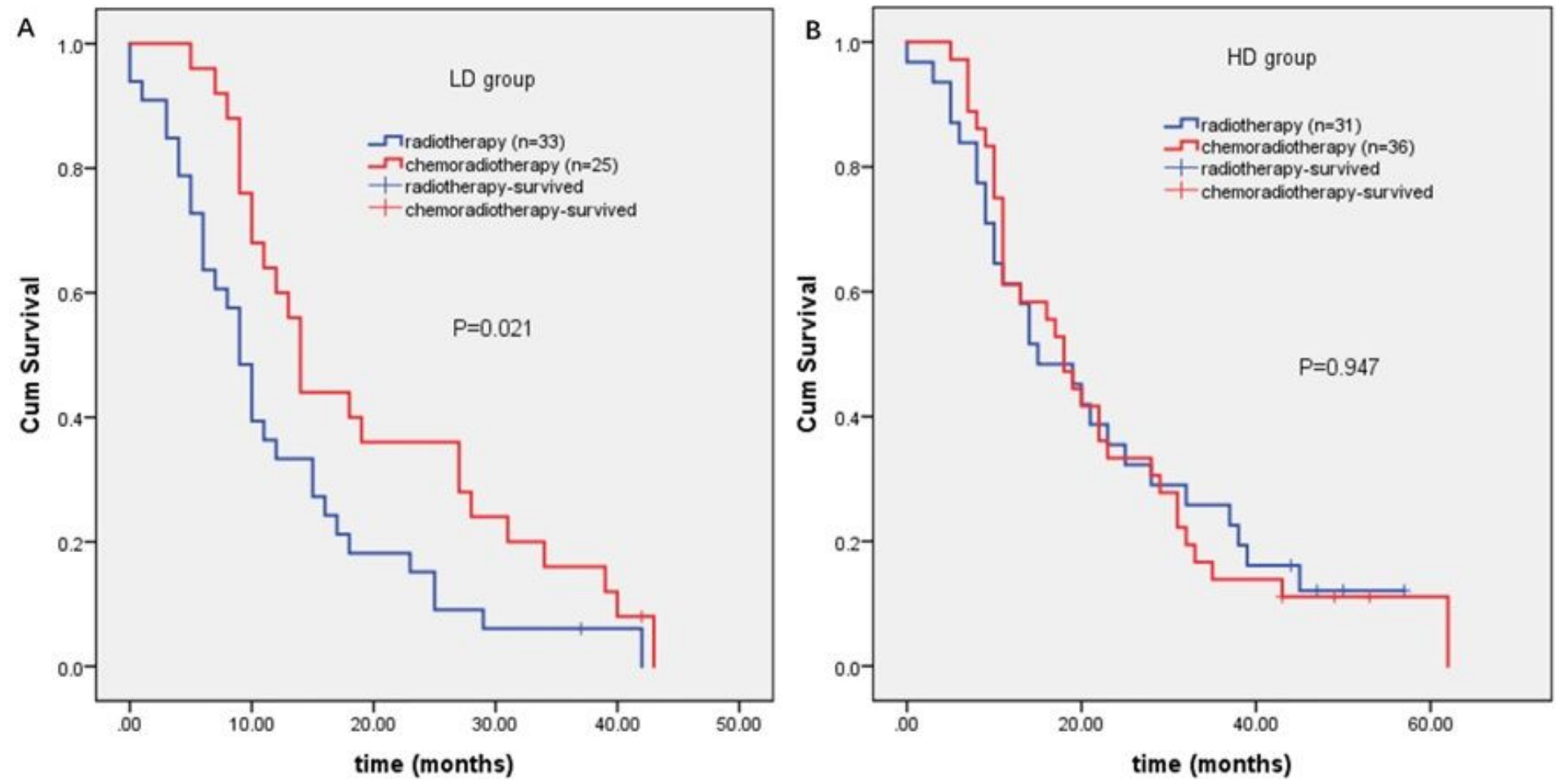

Figure 4 
A Survival curve of patients in low-dose group with chemoradiotherapy and re-irradiotherapy alone. B Survival curve of patients in high-dose group with chemoradiotherapy and re-irradiotherapy alone. 